Tecno Lógicas

ISSN 0123-7799

Vol. 20, No. 38, pp. 41-53

Enero - junio de 2017

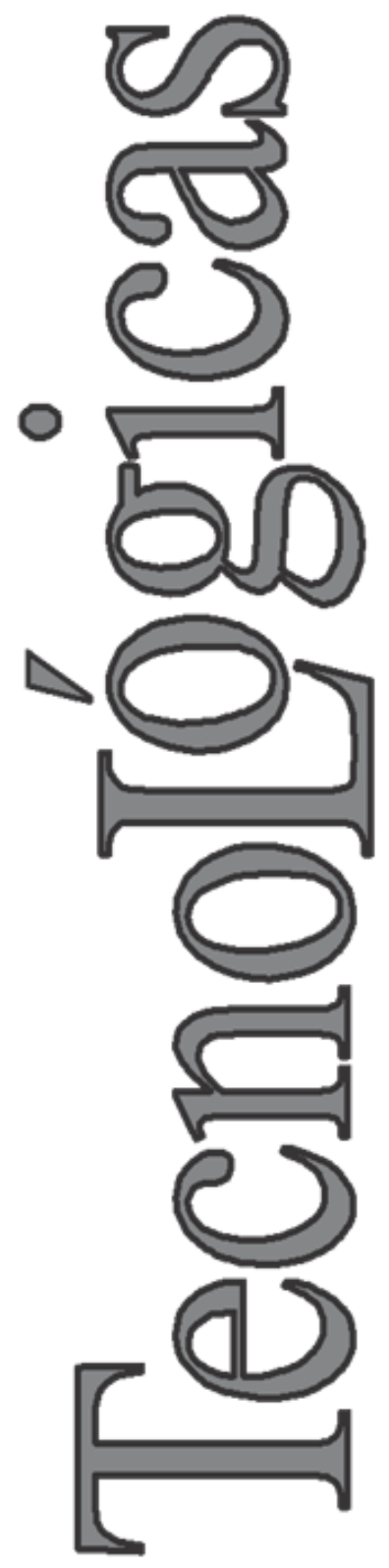

(C) Copyright 2015 por autores y Tecno Lógicas Este trabajo está licenciado bajo una Licencia Internacional Creative Commons Atribución (CC BY)

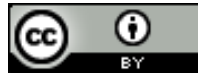

\section{Metodología para la determinación del desplazamiento angular en transformadores trifásicos}

\section{Methodology for determining angular displacement in three-phase transformers}

\author{
Nicolás Muñoz-Galeano ${ }^{1}$, Jesús M. López-Lezama² \\ y Fernando Villada-Duque ${ }^{3}$
}

Recibido: 12 de agosto de 2015, Aceptado: 19 de septiembre de 2016

Cómo citar / How to cite

N. Muñoz-Galeano, J.M. López-Lezama y F. Villada-Duque, "Metodología para la determinación del desplazamiento angular en transformadores trifásicos", Tecno Lógicas, vol. 20, no. 38, pp. 41 - 53 enero-junio, 2017.

1 Doctor en Ingeniería Electrónica, Departamento Ing. Eléctrica, Grupo de Manejo Eficiente de la Energía - GIMEL, Facultad de Ingeniería, Universidad de Antioquia, Medellín - Colombia, nicolas.munoz@udea.edu.co

2 Doctor en Ingeniería Eléctrica, Departamento Ing. Eléctrica, Grupo de Manejo Eficiente de la Energía-GIMEL, Universidad de Antioquia, Medellín-Colombia, jmaria.lopez@udea.edu.co

3 Doctor en Ingeniería Eléctrica, Departamento Ing. Eléctrica, Grupo de Manejo Eficiente de la Energía-GIMEL, Universidad de Antioquia, Medellín-Colombia, fernando.villada@udea.edu.co 


\section{Resumen}

Este artículo presenta una metodología para la determinación del desplazamiento angular (conexiones o grupos horarios (coloquial)) en transformadores trifásicos. La metodología propuesta sirve para determinar el desplazamiento angular si se conocen las conexiones o si se desea determinado desplazamiento angular también poder obtener las conexiones. Si bien la conexión de transformadores trifásicos requiere el conocimiento del desplazamiento angular, la literatura técnica no reporta una metodología o procedimiento riguroso explícito para determinarlas. Sin embargo, existen numerosas aplicaciones en las áreas de ingeniería e investigación que requieren el conocimiento de la conexión y el desplazamiento angular. El artículo presenta la metodología para determinar las conexiones $Y_{y}, Y_{d}, D_{y}, D_{d}, Z y$ y $Z_{d}$; que incluye las aplicaciones de ingeniería más relevantes. La metodología propuesta se puede aplicar de forma indiferente tanto para transformadores comerciales como no comerciales o para transformadores de potencia o de distribución.

\section{Palabras clave}

Desplazamiento angular, conexiones, transformadores trifásicos, fasores de tensión y de corriente, método gráfico.

\section{Abstract}

This paper presents methodology for determining angular displacement (connections or vector group (colloquial)) in three-phase transformers. The proposed methodology is useful to determine the angular displacement if connections are known or the connections if angular displacement is known. Even though connection of three-phase transformers requires knowledge of angular displacement; the technical literature does not report a rigorous methodology or procedure for their explicit step by step determination. However, there are numerous applications in the areas of engineering and research that use time group connections. This paper presents the methodology for determining the $Y_{y}, Y_{d}, D_{y}, D_{d}, Z y$ and $Z_{d}$ connections; including the use of time groups in some relevant engineering applications. The proposed methodology can be applied for commercial and noncommercial transformers, and also for power and distribution transformers.

\section{Keywords}

Angular displacement, connections, three-phase transformers, voltage and current phasors, graphic method. 


\section{INTRODUCCIÓN}

En este artículo se propone una metodología para la determinación del desplazamiento angular en transformadores trifásicos. Los autores en [1] presentan una metodología para determinar las conexiones de los transformadores, sin embargo, no es posible observar de forma directa el desplazamiento angular entre las tensiones del primario y del secundario, tampoco presentan algún paso que permita distinguir la conexión de acuerdo con la polaridad del transformador. Usualmente, los ingenieros, para saber las conexiones que tienen que hacer, buscan en algún sitio tablas que les sirven de guía. Las tablas están publicadas en internet, pero no indican el procedimiento para obtener la conexión, normalmente no verifican si la conexión del desplazamiento angular deseado está correcta. Recientemente debido a la dificultad para obtener la conexión y desplazamiento angular, los autores en [2] presentan un sistema de adquisición de señales que permite obtener en línea el desplazamiento angular y las conexiones de los transformadores; sin embargo, no todos los ingenieros, cuando necesitan obtener el desplazamiento angular pueden acceder al sistema de adquisición.

La metodología propuesta brinda herramientas para determinar el desplazamiento angular de forma rápida y sencilla, presentando un método de 5 pasos generalizados que sirve para obtener el desplazamiento angular para cualquier tipo de conexión. Este trabajo realiza un ejercicio fasorial riguroso y muestra dependiendo de la conexión las particularidades que resultan producto de todas las posibles conexiones considerando la polaridad de las bobinas. La metodología considera dentro de sus pasos la conexión del primario y del secundario, pudiéndose observar de forma directa el desplazamiento angular entre la tensión del primario y la tensión del secundario. La metodología puede incluirse en los cursos o seminarios de transforma- dores para ingenieros electricistas o profesiones afines contribuyendo a la mejora de su formación.

Una de las aplicaciones habituales que requiere la determinación del desplazamiento angular es la conexión de transformadores en paralelo. Los transformadores en paralelo deben conectarse con el mismo desplazamiento angular para evitar corrientes peligrosas entre los devanados que se conectan en paralelo. Revisando la literatura técnica se encontró que existe gran cantidad de aplicaciones que requieren la determinación del desplazamiento angular. Estas aplicaciones se resumen a continuación: en [3] se presenta un método de compensación de corrientes reactivas, corrientes armónicas y corrientes secuencia negativa. Se utiliza un transformador de conexión $Y d 11$ para reducir las componentes de secuencia cero. En [4] se propone el uso de dos transformadores en paralelo para la mitigación de armónicos de corriente en inversores trifásicos. Uno de los transformadores tiene conexión $D y$ y el otro tiene conexión $\mathrm{Dz}$. Ambos transformadores deben tener el mismo desfase entre tensiones primarias y secundarias para poder conectarlos en paralelo. La conexión en delta del primario evita que los armónicos de corriente de tercer orden fluyan hacia la red eléctrica, la conexión en estrella permite reducir los armónicos de corriente pares y la conexión zig-zag reduce las componentes de corriente de secuencia cero, producto del desequilibrio. En [5] se utiliza un trasformador con conexión $D y$ en inversores trifásicos, los autores indican que utilizan la conexión $D y$ debido a que tiene como ventajas la reducción de los armónicos de tercer orden y una mejor relación de voltaje de línea con respecto a la conexión $Y y$, lo que reduce potencialmente su costo. En [6] se indican las desventajas de la conexión en estrella en algún devanado del transformador. La conexión estrella con neutro sin aterrizar tiene un aislamiento del sistema, pero ante cargas desbalanceadas presenta un voltaje tierra neutro. La conexión estrella 
con neutro aterrizado no presenta desbalance en el punto neutro, pero se pierde el aislamiento entre el transformador y el sistema. En [7], [8], [9] y [10] se utiliza el transformador en conexión zig-zag como filtro de secuencia cero para aplicaciones de electrónica de potencia. En [11] se propone un método para disminuir las pérdidas de potencia en los transformadores con conexiones en delta. Una solución consistía en usar la conexión $Y y$, esto debido a que no produce cambios de fase en los voltajes de salida y necesita poco aislamiento. Aunque indica que la conexión $Y y$ no debe usarse ante cargas no lineales porque no permite la reducción en la red de los armónicos de corriente. En [12] se indica que las conexiones $Y d$ y $D y$ tienen como ventaja con respecto a las conexiones $Y y$ y $D d$ el incremento en la relación de transformación, permitiendo la reducción del estrés de corriente y de voltaje. En [13] se presentan varios esquemas de conexión para conversores DC/DC con modulación de pulso usando transformadores con diferentes conexiones. Como uno de los resultados se resalta el incremento en las pérdidas en el cobre cuando se utiliza la conexión $Y d$; mientras que el transformador con conexión $D d$ presenta el incremento en las pérdidas en el hierro. En [14] se indica que los transformadores $Y y, D y$ son útiles en sistemas balanceados pero ante cargas monofásicas inherentemente desbalanceadas, las corrientes de retorno por el conductor neutro pueden generar un desbalance en el sistema de distribución. Como solución se proponen varios métodos de compensación pasiva usando reactores que bloquean las componentes de secuencia cero ZSBR (Zero Sequence Blocking Reactor) y transformadores con conexiones $Y d$ y zig-zag que comparten la propiedad de mitigar la corriente que circula por el conductor neutro. La conexión $Y d$ permite la reducción de los armónicos de tercer orden; mientras que el filtro reduce los armónicos que la conexión del transformador no pueda reducir.
En la siguiente sección se explica la metodología para determinar el desplazamiento angular en los transformadores. La metodología propuesta puede aplicarse a cualquier transformador trifásico independiente de la conexión, sin embargo, se incluyen algunos ejemplos que permiten observar las particularidades del método para cada conexión. Al final de esta sección, se presenta una tabla que resume la aplicación de la metodología para las conexiones más comunes.

\section{METODOLOGíA}

\subsection{Metodología para la determinación del desplazamiento angular}

La conexión determina el desplazamiento angular entre el voltaje primario y el voltaje secundario. El desplazamiento angular entre el voltaje primario y el voltaje secundario depende de la conexión del transformador, de la carga y de la impedancia de las bobinas. El desplazamiento angular provocado por la conexión del transformador es llamado coloquialmente grupo horario debido a que siempre es múltiplo de $30^{\circ}$. Es por esto que, al realizar el diagrama fasorial, los fasores de voltaje del primario y secundario se ubican sobre las horas del reloj de pared.

En este artículo, a las terminales del primario se le asignan letras mayúsculas $(\mathrm{A}, \mathrm{B}, \mathrm{C}, \mathrm{N})$ para las fases y el neutro, mientras que a las terminales del secundario se le asignan letras minúsculas $(\mathrm{a}, \mathrm{b}, \mathrm{c}$, n). Similarmente, para denotar los fasores de voltaje del primario se utilizan subíndices con las letras (A, B, C); mientras que para los fasores de voltaje del secundario se utilizan subíndices con las letras (a, b, c). El fasor de voltaje del primario de la fase $\mathrm{A}\left(V_{A}\right)$ es la referencia para determinar el desplazamiento angular y se ubica sobre el eje positivo de las ordenadas, asignándole en el reloj las cero horas. El fasor de 
voltaje del secundario de la fase a $\left(V_{a}\right)$ se ubica sobre cualquiera de las 12 horas del reloj estableciendo la hora de la conexión. La metodología para ser aplicada solo requiere la medición de la polaridad de las bobinas, ya que la metodología tras observar las conexiones permite determinar el desplazamiento angular. La metodología también permite establecer a partir del desplazamiento angular deseado la conexión que se debe realizar, solo se deben seguir los pasos de atrás hacia adelante.

El transformador, en su aplicación más común, se utiliza para cambiar el nivel de voltaje. Para hacerlo se aprovecha el principio de inducción electromagnética de Faraday: un flujo magnético variable en el tiempo genera en una bobina un voltaje inducido que depende del número de vueltas de la bobina y de la variación del flujo. Es por esto que, al energizar con una tensión alterna (senoidal) en el primario de un transformador, se produce una corriente que magnetiza el núcleo provocando un flujo alterno. El flujo alterno, al pasar por el devanado secundario, genera un voltaje inducido proporcional al voltaje del devanado primario. El transformador está compuesto por bobinas magnéticamente acopladas. Para bobinas de una misma rama de transformador, las fem de las bobinas están en fase si las bobinas se encuentran arrolladas en un mismo sentido. Si las bobinas están arrolladas en sentidos contrarios, las fem están en contrafase, es decir tienen un desfase de $180^{\circ}$. La metodología propuesta no incluye un procedimiento para determinar la polaridad de las bobinas, sin embargo la polaridad de las bobinas puede obtenerse fácilmente conectando el transformador como autotransformador y verificando si las bobinas son aditivas o sustractivas [15].

La metodología propuesta para determinar el desplazamiento angular consiste en un método gráfico de 5 pasos y se describe a continuación:

Paso 1: Establecimiento de la referencia de los fasores: la referencia es el fasor de voltaje de alimentación de la fase A en el primario del transformador. Se dibuja el fasor de voltaje de alimentación de la fase A que es la referencia de los ángulos y se ubica sobre el eje positivo de las abscisas marcando las cero horas en el reloj de pared. Luego se dibujan los fasores de voltaje para las fases B y C en secuencia positiva. El voltaje primario de la fase B está desfasado $-120^{\circ}$ marcando las 4 horas. Mientras que el voltaje de la fase $\mathrm{C}$ está desfasado $120^{\circ}$ marcando las 8 horas. El método propuesto requiere que la red eléctrica tenga secuencia positiva que es la secuencia de las fases establecida para las tensiones de todos los sistemas eléctricos de potencia, en el caso de que el sistema eléctrico tenga un sistema de tensiones de secuencia negativa (conexión errónea) el método propuesto no podría utilizarse tal y como se plantea en este artículo. Este paso siempre es el mismo no dependiendo de la conexión o del tipo de transformador, por lo que la descripción de los ejemplos de la metodología se referirá para este paso siempre a la Fig. 1.

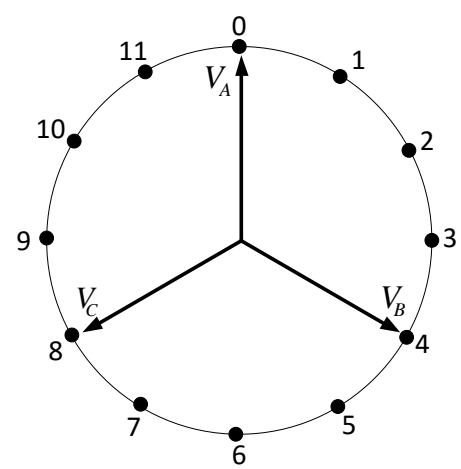

Fig. 1. Paso 1 de la metodología, referencia de voltaje. Fuente: Autores.

Paso 2: Determinación del voltaje de las bobinas del primario: no siempre el voltaje en las terminales del transformador es la fem que se induce en sus devanados, ya que depende de su conexión Y o D. Para la conexión $\mathrm{Y}$, el voltaje de alimentación en las terminales A, B y C coincide con la fem que se establece en cada bobina. Para la conexión $\mathrm{D}$, la fem que se establece en cada bobina del transformador proviene de un 
voltaje compuesto aplicado que puede obtenerse como la resta fasorial entre el voltaje que se aplica sobre la terminal positiva de la bobina (la terminal del punto) y el voltaje que se aplica sobre la terminal de negativa de la bobina.

Paso 3: Determinación de la fem inducida en las bobinas del secundario: debido a que las bobinas del primario y secundario de cada fase comparten el flujo de las columnas del núcleo magnético, los fasores de voltaje que se establecen en las bobinas secundario son proporcionales y pueden estar en fase o contrafases con los fasores de voltaje del primario. Si están en fase, es porque los devanados primario y secundario están arrollados en el mismo sentido y los fasores de voltaje del secundario deben dibujarse en el mismo sentido de los fasores del primario. Si están en contrafase, es porque los devanados primario y secundario están arrollados en sentido contrario y los fasores de voltaje del secundario se dibujan en sentido contrario a los fasores de voltaje del primario. Dibujar en sentido contrario los fasores equivale a multiplicar por - 1 para cambiar la fase, por ejemplo, si el fasor de voltaje del primario tiene fase de $0^{\circ}$ el fasor de voltaje inducido en el secundario tiene fase de $180^{\circ}$. Debido a que el desplazamiento angular está incluido al dibujar los fasores del secundario en contrafase, hay que prestar especial atención no cambiando la polaridad en el esquemático si no cambiando solamente el dibujo. Para determinar el desplazamiento angular es irrelevante la relación de transformación, aunque para propósitos ilustrativos se supondrá que los voltajes del secundario del transformador son menores que los voltajes del primario. Para distinguir las fem que se inducen en las bobinas del secundario del transformador se utilizarán para los fasores del secundario los mismos subíndices de los fasores de las bobinas del primario, pero adicionalmente se utilizará como superíndice la comilla. Por ejemplo, si la fem que se establece en una de las bobinas del primario es $V_{A C}$, en la bobina co- rrespondiente del secundario se inducirá una fem $V_{A C}^{\prime}$.

Paso 4: Obtención de los voltajes en las terminales a, b y c del secundario: Los voltajes que se establecen en las terminales a, b, y c del transformador dependen de la conexión de los devanados. Si la conexión está en estrella, los voltajes sobre las terminales son los mismos que las fems inducidas en las bobinas del secundario del transformador. Si la conexión está en triangulo o zig-zag debe realizarse la suma fasorial para poder extraer los fasores de voltaje sobre las terminales.

Paso 5: Superposición de los fasores de voltaje $V_{A}, V_{B}$ y $V_{C}$ con los fasores de voltaje $V_{a}, V_{b}$ y $V_{c}$ para determinar el grupo horario: La conexión del primario se denota mediante las letras en mayúscula $Y$ o $D$, dependiendo del caso. La conexión del secundario se denota mediante las letras en minúscula $y, d$ ó $z$, dependiendo del caso. La hora en el reloj de pared hacia donde apunta el fasor $V_{a}$ determina el desplazamiento angular de la conexión.

\section{RESULTADOS: EJEMPLOS DE APLICACIÓN}

Las siguientes subsecciones muestran la metodología aplicada a las conexiones más comunes que se presentan en los sistemas de potencia. Para mostrar mejor las particularidades de la metodología, las siguientes subsecciones incluyen ejemplos que la usan. La primera subsección aplica la metodología para determinar el desplazamiento angular de las conexiones $Y y 0 \mathrm{y}$ Yy6. La segunda subsección aplica la metodología para determinar el desplazamiento angular de las conexiones Dyx $(\mathrm{x}=1,5,7,11)$. La tercera subsección aplica la metodología para determinar el desplazamiento angular de las conexiones $D d x$ $(\mathrm{x}=0,2,4,6,8,10)$. La cuarta subsección presenta una tabla que incluye el desplazamiento angular de las conexiones $Y d x$ 
$(x=1,5,7,11)$ y algunas conexiones zig-zag (Yzx y Dzx con $x$ entero desde 0 hasta 11).

\section{Conexiones Yy0 y Yy6}

Las conexiones $Y y 0$ y $Y y 6$ se utilizan en aplicaciones que requieren del neutro, tanto en el primario como en el secundario. Se utiliza la conexión $Y$ cuando se tienen cargas desequilibradas que generan una corriente de retorno por el neutro. Para la conexión $Y$ con neutro, se deben diferenciar las tensiones en los devanados de acuerdo a donde se mida; así que: 1) Se denomina tensión fase neutro o simplemente tensión de fase a la tensión que se establece entre la fase y el neutro. 2) Se denomina tensión línea a línea o simplemente tensión de línea a la tensión que se establece entre las fases de las bobinas del transformador. Para las conexiones $Y y$ todas las corrientes de la carga en el secundario se reflejan en el lado primario. La Tabla 1 describe la metodología para las conexiones $Y y 0$ y $Y y 6$.

Metodología para la conexión Yy0 (segunda fila de la Tabla 1):

Paso 1: Establecimiento de la referencia de los fasores: Ver Fig 1.

Paso 2: Determinación del voltaje de las bobinas del primario: la conexión $Y$ en el primario indica que los voltajes que se establecen en las bobinas del primario coinciden con los fasores $V_{A}, V_{B}$ y $V_{C}$, ver fasores de la cuarta columna de la Tabla 1.

Paso 3: Determinación del voltaje de las bobinas del secundario: debido a que las bobinas del primario y del secundario tienen la misma polaridad, los fasores $V_{A}^{\prime}, V_{B}^{\prime} \mathrm{y}$ $V_{C}^{\prime}$ tienen el mismo sentido que los fasores $V_{A}, V_{B}$ y $V_{C}\left(V_{A}^{\prime}, V_{B}^{\prime}\right.$ y $V_{C}^{\prime}$ son los fasores de la quinta columna de la Tabla 1 ).

Paso 4: Obtención de los voltajes en las terminales a, b y c del secundario: los fasores $V_{a}, V_{b} \mathrm{y} V_{c}$ coinciden respectivamente con $V_{A}^{\prime}, V_{B}^{\prime}$ y $V_{C}^{\prime}$ debido a que el secundario está en $y$. Ver sexta columna de la Tabla 1.

Paso 5: Superposición de los fasores $V_{A}$, $V_{B}$ y $V_{C}$.con los fasores de voltaje $V_{a}, V_{b}$ y $V_{c}$. (Ver séptima columna de la Tabla 1). El grupo horario es $Y y 0$.

Para la obtención del desplazamiento angular para la conexión Yy6 (tercera fila de la Tabla 1), la descripción de los pasos 1,2, 4 y 5 coinciden con la descripción de los pasos 1,2, 4 y 5 de la conexión $Y y 0$, por lo que no serán mencionados nuevamente. El paso 3 para la conexión Yy6 es:

Paso 3: Determinación del voltaje de las bobinas del secundario: debido a que las bobinas del primario y del secundario tienen la polaridad contraria, los fasores $V_{A}^{\prime}$, $V_{B}^{\prime}$ y $V_{C}^{\prime}$ tienen el sentido contrario que los fasores $V_{A}, V_{B}$ y $V_{C} . V_{A}^{\prime}, V_{B}^{\prime}$ y $V_{C}^{\prime}$ son los fasores de la quinta columna de la Tabla 1.

Conexiones $Y d x(x=1,7,5$ y 11$)$

Las conexiones $Y d x$ corresponden a transformadores que tienen conexión $Y$ en el primario y conexión delta en el secundario. Una de las aplicaciones más importantes para el grupo de conexión $Y d$ es el transformador que se conecta en la salida del generador. En el primario o lado de la $Y$, se conecta el generador que requiere el neutro como camino de retorno para las corrientes de falla cuando ocurren cortocircuitos. En el lado secundario o lado de la delta, se conecta la línea de transmisión encargada de transmitir la potencia desde la generación hacia las cargas. La conexión delta permite la transmisión de potencia con solo tres conductores evitando el uso del conductor del neutro por varios miles de kilómetros de distancia. El desfase se debe a la conexión del secundario que está en delta. La Tabla 2 describe la metodología para las conexiones $Y d x(x=1,7,5 y 11)$. 
Tabla 1. Metodología aplicada para los grupos horarios Yy0 y Yy6. Fuente: Autores.

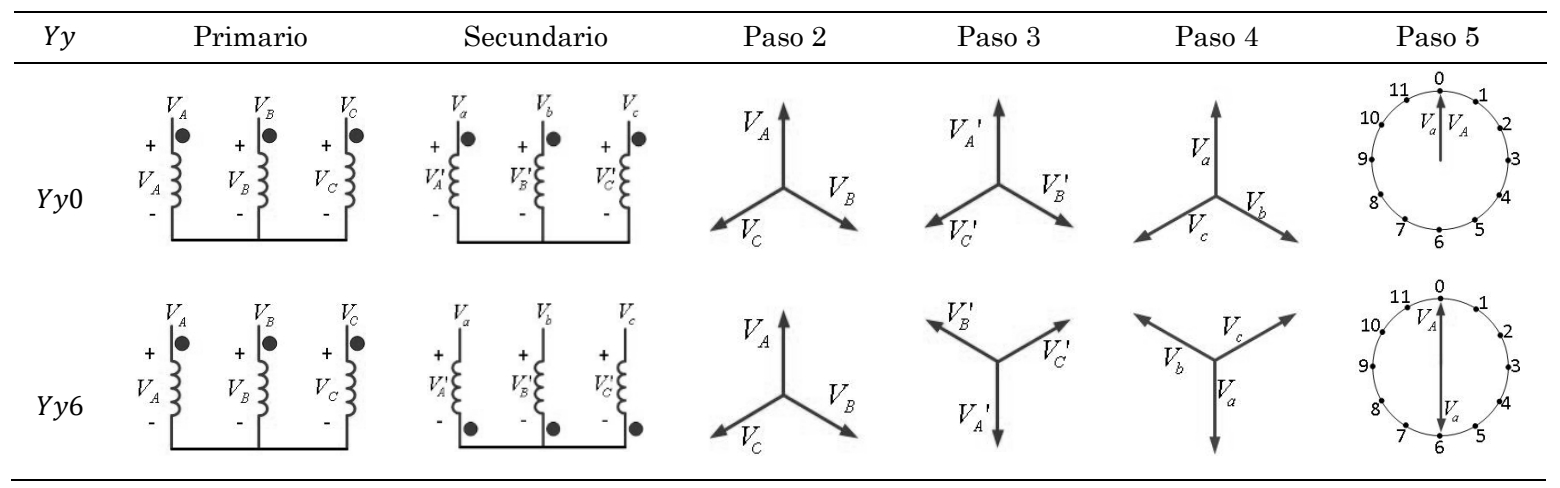

Tabla 2. Metodología aplicada para las conexiones $Y d x(x=1,7,5$ y 11). Fuente: Autores.

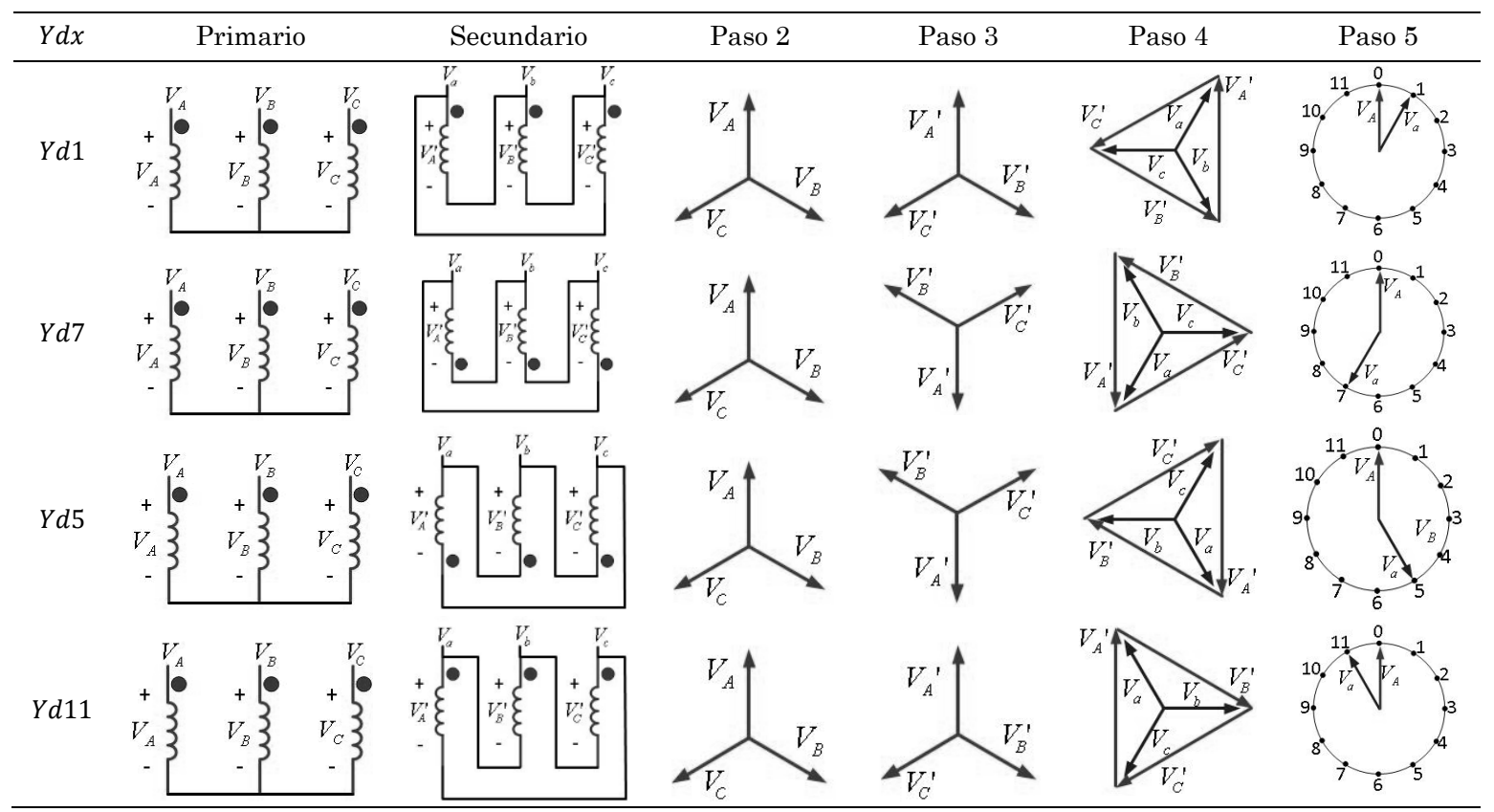

Como ejemplo, a continuación se describirá el procedimiento para la obtención de la conexión $Y d 1$ (ver fila 2).

Paso 1: Establecimiento de la referencia de los fasores: Ver Fig. 1.

Paso 2: Determinación del voltaje de las bobinas del primario: la conexión $Y$ en el primario indica que los voltajes que se establecen en las bobinas del primario coinciden con los fasores $V_{A}, V_{B}$ y $V_{C}$. Ver columna 4.

Paso 3: Determinación del voltaje de las bobinas del secundario: debido a que las bobinas del primario y del secundario tienen la misma polaridad, los fasores $V_{A}^{\prime}, V_{B}^{\prime} \mathrm{y}$ $V_{C}^{\prime}$ tienen el mismo sentido que los fasores
$V_{A}, V_{B}$ y $V_{C}\left(V_{A}^{\prime}, V_{B}^{\prime}\right.$ y $V_{C}^{\prime}$ son los fasores de la columna 5).

Paso 4: Obtención de los voltajes en las terminales a, b y c del secundario: debido a que el secundario está en delta, los voltajes sobre las bobinas en el secundario $\left(V_{A}^{\prime}, V_{B}^{\prime}\right.$, $\left.V_{C}^{\prime}\right)$ son voltajes compuestos. El voltaje $V_{A}^{\prime}$ es la resta entre $V_{a}$ y $V_{b}\left(V_{A}^{\prime}=V_{a}-V_{b}\right)$; el voltaje $V_{B}^{\prime}$ es la resta entre $V_{b}$ y $V_{c}\left(V_{B}^{\prime}=\right.$ $V_{b}-V_{c}$ ); y El voltaje $V_{C}^{\prime}$ es la resta entre $V_{c} \mathrm{y}$ $V_{a}\left(V_{B}^{\prime}=V_{b}-V_{c}\right)$. Los voltajes $V_{a}, V_{b}$ y $V_{c}$ son los voltajes en las terminales a, b y c del secundario, gráficamente pueden ser obtenidos si se hace la suma fasorial de $V_{A}^{\prime}, V_{B}^{\prime} \mathrm{y}$ $V_{C}^{\prime}$ (ver fasores de la columna 6). De la suma fasorial de $V_{A}^{\prime}, V_{B}^{\prime}$ y $V_{C}^{\prime}$, puede verse que 
generan un triángulo equilátero. Desde el centro del triángulo se trazan los fasores $V_{a}, V_{b}$ y $V_{c}$ que verifican las ecuaciones $\left(V_{A}^{\prime}=V_{a}-V_{b}, V_{B}^{\prime}=V_{b}-V_{c}, V_{C}^{\prime}=V_{c}-V_{a}\right)$.

Paso 5: Superposición de los fasores $V_{A}$, $V_{B}$ y $V_{C}$.con los fasores de voltaje $V_{a}, V_{b}$ y $V_{c}$. (Ver séptima columna de la tabla 2). El grupo horario es $Y d 1$.

\section{Conexiones Dd0 y Dd6}

Las conexiones $D d$ corresponden a los transformadores que tienen conexión $D$ en el primario y conexión d en el secundario. Una de las aplicaciones más importantes para el grupo de conexión $D d$ es la interconexión entre sistemas de transmisión $\mathrm{y}$ subtransmisión, ambos sistemas requieren la conexión delta para ahorrar el cable del neutro. Si el primario y secundario tienen la misma polaridad, el desfase entre $\mathrm{V}_{\mathrm{A}}$ y $V_{a}$ es de $0^{\circ}(D d 0)$. Si el primario y secundario tienen la polaridad contraria, el desfase entre $V_{\mathrm{A}}$ y $V_{a}$ es de $180^{\circ}(D d 6)$. La Tabla 3 describe la metodología para las conexiones Dd0 y $D d 6$.

Como ejemplo, a continuación se describirá el procedimiento para la obtención del desplazamiento angular de la conexión Dd6. (ver fila 3).

Paso 1: Establecimiento de la referencia de los fasores: Ver Fig. 1.

Paso 2: Determinación del voltaje de las bobinas del primario: Para la conexión $D$ en el primario, se establecen voltajes compuestos en cada rama del transformador. Para la bobina 1 se establece la tensión $V_{A C}$ $\left(V_{A C}=V_{A}-V_{C}\right)$; esto debido a que se alimenta la terminal positiva de la bobina 1 con $V_{A}$, mientras que la terminal negativa es alimentada con $V_{C}$. Similarmente ocurre con la bobina 2 estableciéndose $V_{B A}\left(V_{B A}=\right.$ $\left.V_{B}-V_{A}\right)$ y con la bobina 3 estableciéndose $V_{C B}\left(V_{C B}=V_{C}-V_{B}\right)$. Los fasores $V_{A C}, V_{B A} \mathrm{y}$ $V_{C B}$ están dibujados en la columna 4.
Paso 3: Determinación del voltaje de las bobinas del secundario: la polaridad contraria indica que los fasores $V_{A C}^{\prime}, V_{B A}^{\prime}$ y $V_{C B}^{\prime}$ están en contrafase con respecto a $V_{A C}, V_{B A}$ y $V_{C B}$; es por esto que los fasores $V_{A C}^{\prime}, V_{B A}^{\prime} \mathrm{y}$ $V_{C B}^{\prime}$ tienen sentido contrario con respecto a $V_{A C}, V_{B A}$ y $V_{C B}$ (ver fasores de la columna 5). Los fasores $V_{A C}^{\prime}, V_{B A}^{\prime}$ y $V_{C B}^{\prime}$ pueden dibujarse indiferentemente en estrella o triángulo, en este caso se ha elegido dibujarlos en triángulo por la conexión delta del secundario.

Paso 4: Obtención de los voltajes en las terminales a, b y c del secundario: debido a que el secundario está en delta, los voltajes sobre las bobinas en el secundario $\left(V_{A C}^{\prime}, V_{B A}^{\prime}\right.$ y $V_{C B}^{\prime}$ ) son también voltajes compuestos en el secundario del transformador. De la figura en la columna 6 puede verse que: $\mathrm{El}$ voltaje $V_{A C}^{\prime}$ es la resta entre $V_{a}$ y $V_{c}\left(V_{A C}^{\prime}=\right.$ $V_{a}-V_{c}$ ); el voltaje $V_{B A}^{\prime}$ es compuesto porque es la resta entre $V_{b}$ y $V_{a}\left(V_{B A}^{\prime}=V_{b}-V_{a}\right)$; y El voltaje $V_{C B}^{\prime}$ es la resta entre $V_{c}$ y $V_{b}\left(V_{C B}^{\prime}=\right.$ $\left.V_{c}-V_{b}\right)$. Los voltajes $V_{a}, V_{b}$ y $V_{c}$ son los voltajes en las terminales a, b y c del secundario, gráficamente pueden ser obtenidos si se hace la suma fasorial de $V_{A C}^{\prime}, V_{B A}^{\prime}$ y $V_{C B}^{\prime}$. De la suma fasorial de $V_{A C}^{\prime}, V_{B A}^{\prime}$ y $V_{C B}^{\prime}$, puede verse que generan un triángulo equilátero. Desde el centro del triángulo se trazan los fasores $V_{a}, V_{b}$ y $V_{c}$ que verifican las ecuaciones $\left(V_{A C}^{\prime}=V_{a}-V_{c}, V_{B A}^{\prime}=V_{b}-V_{a}\right.$, $\left.V_{C B}^{\prime}=V_{c}-V_{b}\right)$.

Paso 5: Superposición de los fasores $V_{A}$, $V_{B}$ y $V_{C}$.con los fasores de voltaje $V_{a}, V_{b}$ y $V_{c}$. (Ver séptima columna de la Tabla 2). La conexión es $D d 6$.

\section{Conexiones Dyx $(x=1,7,11$ y 5$)$}

Las conexiones Dyx suelen usarse en sistemas de distribución. La conexión $D$ del primario atrapa en la delta las componentes del tercer armónico de corriente que pueda tener la carga. 
Tabla 3. Metodología aplicada para las conexiones $D d 0$ y $D d 6$. Fuente: Autores.

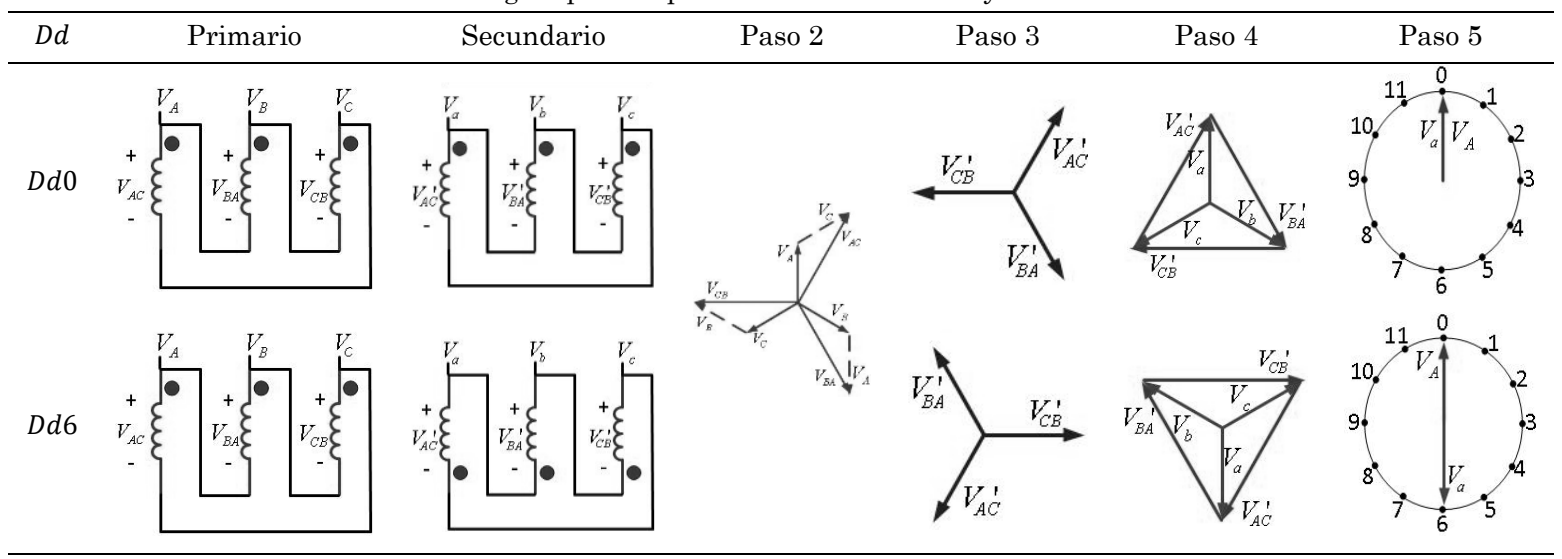

La conexión en y del secundario propicia camino de retorno para la corriente que va por el neutro en la instalación. La Tabla 4 describe la metodología para las conexiones $\operatorname{Dyx}(x=1,7,11$ y 5$)$.

\section{Conexiones Yzx y Dzx (x entero desde 0} hasta 11).

Las conexiones $Y z x$ y $D z x$ suelen usarse para aterrizar líneas de transmisión, debido a que el neutro de la conexión zig-zag propicia un camino para las corrientes de secuencia cero o armónicos de corriente de tercer orden. La conexión zig-zag también propicia la eliminación de armónicos de tensión de tercer orden disminuyendo el desequilibrio de tensión que puede generarse en líneas de transmisión. Debido a la conexión zig-zag, existen gran cantidad de combinaciones. Por lo que, las Tablas 5 y 6 solo describe la metodología para las conexiones $Y z 11$ y $D z 2$, respectivamente. Otras conexiones que incluyen devanados en conexión zig-zag pueden obtenerse fácilmente siguiendo la metodología propuesta.

La metodología propuesta también permite obtener la conexión de los transformadores si se parte del desplazamiento angular. Fácilmente, empezando desde el último paso de la metodología y ejecutando los pasos hasta llegar al primero es posible obtener la conexión de las bobinas del transformador. Para iniciar la metodología de atrás hacia adelante, además de conocer el desplazamiento angular, se debe conocer o elegir qué tipo de conexión deben tener los devanados primarios $(Y$ o $D)$ y secundarios $\left(\begin{array}{lll}y & 0 & d\end{array}\right)$. Las particularidades de la metodología cuando se aplica de atrás hacia adelante se detallan a continuación:

Paso 5: El paso 5 requiere el conocimiento del desplazamiento angular deseado, se deben dibujar los fasores a los que se pretende llegar.

Paso 4: El desplazamiento angular depende de la conexión del devanado. Si la conexión es $y$, los fasores representados en el paso 5 son los mismos fasores que deben dibujarse en el paso 4; debiendo en este paso conectarse los devanados en $y$. Si la conexión es $d$ y el fasor del secundario deseado esta desplazado en el mismo sentido del sentido de las manecillas del reloj se debe conectar: 1) El positivo del devanado a con el negativo del devanado c, 2) el positivo del devanado b con el negativo del devanado a $\mathrm{y}, 3$ ) el positivo del devanado c con el negativo del devanado b. Si la conexión es $d$ y el fasor del secundario deseado esta desplazado en sentido contrario con respecto al sentido de las manecillas del reloj se debe conectar: 1) El positivo del devanado a con el negativo del devanado $b$, 2) el positivo del devanado b con el negativo del devanado c y, 3) el positivo del devanado c con el negativo del devanado a.

Paso 3: Si el desplazamiento angular deseado esta sobre la semicircunferencia superior del reloj de pared, indica que la 
polaridad del transformador es positiva; de lo contrario, si el desplazamiento angular deseado se ubica sobra la semicircunferencia inferior del reloj de pared, entonces la polaridad es negativa.

Paso 2: Al igual que el paso 4, el desplazamiento angular depende de la conexión. Si la conexión es $Y$, los fasores representados en el paso 3 son los mismos fasores que deben dibujarse en el paso 2 . Si la conexión es $D$ y el fasor del secundario deseado está desplazado en el mismo sentido del sentido de las manecillas del reloj se debe conectar: 1) El positivo del devanado A con el negativo del devanado C, 2) El positivo del devanado $\mathrm{B}$ con el negativo del devanado A y 3) el positivo del devanado $\mathrm{C}$ con el negativo del devanado B. Si la conexión es $D$ y el fasor del secundario deseado está desplazado en sentido contrario con respecto al sentido de las manecillas del reloj se debe conectar: 1) El positivo del devanado A con el negativo del devanado B, 2) El positivo del devanado $\mathrm{B}$ con el negativo del devanado $\mathrm{C} \mathrm{y}, 3$ ) $\mathrm{El}$ positivo del devanado $\mathrm{C}$ con el negativo del devanado A.

Paso 1: Al devolverse en la metodología, los fasores que resultan en las terminales $\mathrm{A}, \mathrm{B}$ y $\mathrm{C}$ deben coincidir con los fasores de la Fig 1.

Tabla 4. Metodología aplicada para las conexiones Dyx $(x=1,7,11$ y 5). Fuente: Autores.

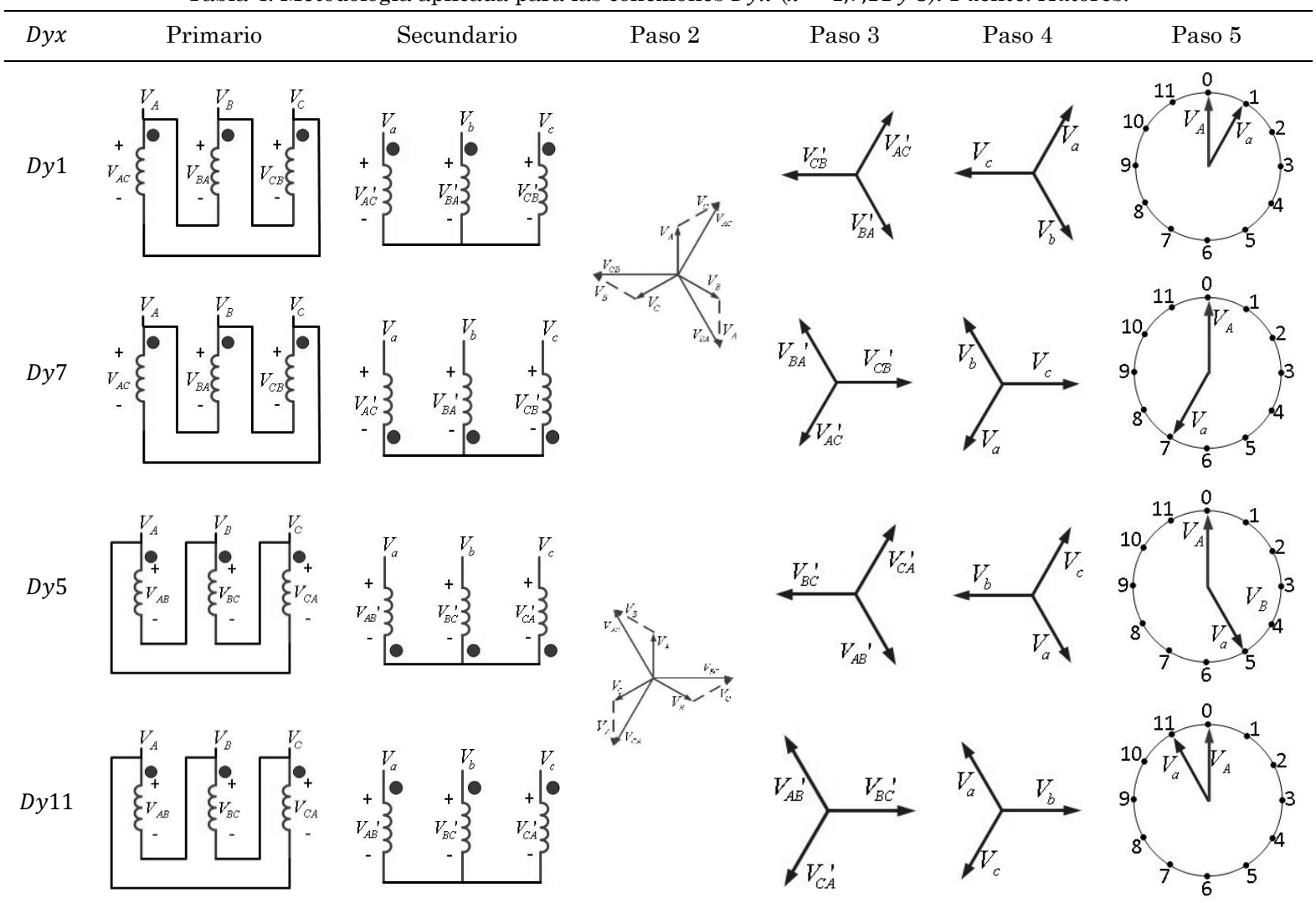


Tabla 5. Metodología para la conexión Yz11. Fuente: Autores.

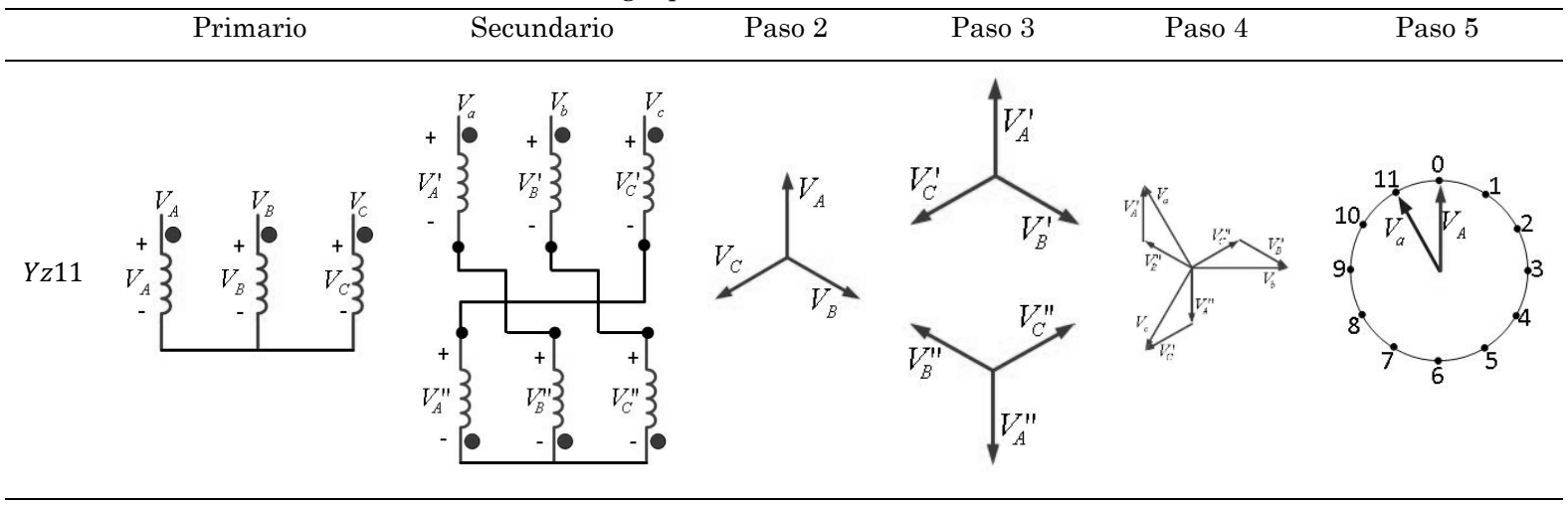

Tabla 6. Metodología para la conexión Dz2. Fuente: Autores.

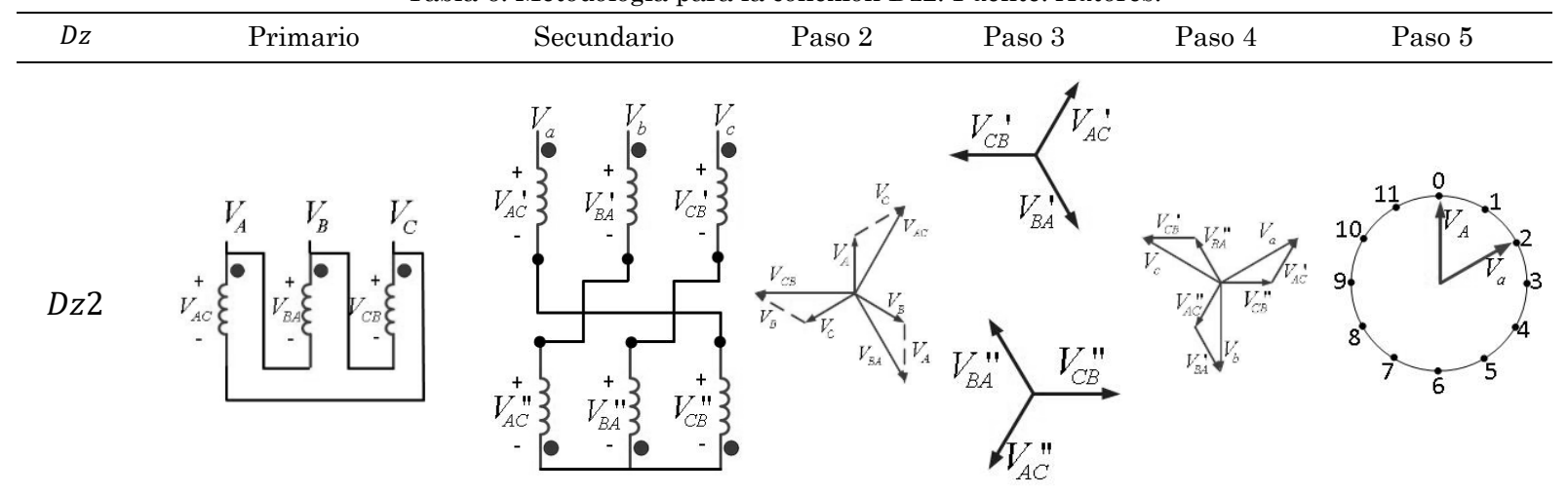

\section{CONCLUSIONES}

El artículo presenta una metodología fácil y sencilla para determinar el desplazamiento angular cuando se conoce la conexión del transformador. Si se sigue la metodología propuesta pueden determinarse tanto el desplazamiento angular de trasformadores comerciales como no comerciales. La metodología incluye cinco pasos gráficos que permiten entender lo que sucede con los fasores de voltaje en cada devanado y en las terminales de conexión. La metodología propuesta puede usarse en los cursos de transformadores para la formación de ingenieros electricistas y profesiones afines.

La metodología propuesta tiene 5 pasos perfectamente definidos y puede aplicarse de forma general a cualquier transforma- dor trifásico, sin embargo, se observan particularidades en cada paso de la metodología que se enuncian a continuación: 1)

la tensión de los devanados en conexión estrella es la tensión en las terminales de las bobinas, 2) los devanados en conexión delta tienen tensiones compuestas y dependen de las terminales conectadas en los extremos de cada bobina, 3) el desfase de los voltajes que se establecen en devanados con conexión delta con respecto a los voltajes sobre sus terminales es de \pm 30 grados, 4) cuando el transformador tiene polaridad contraria, el grupo horario se ubica en la parte inferior del reloj, 5) para los transformadores que incluyen la conexión zigzag existen innumerables combinaciones, la metodología propuesta permite determinarlas todas. 


\section{AGRADECIMIENTOS}

Los autores agradecen a la Universidad de Antioquia (UdeA) por el apoyo de "Sostenibilidad 2016-2017"

\section{REFERENCIAS}

[1] E. Marlés Sáenz, "Metodología generalizada para determinar los grupos de conexión," Energía y Comput., vol. 13, no. 2, 2011.

[2] C. A. Hernández Suárez, V. A. Gómez Saavedra, and R. A. Peña Lote, "Sistema de adquisición de datos para determinar el grupo de conexión en el transformador trifásico DL1080," Tecnura, vol. 18, no. 42, pp. 65-77, 2014

[3] W. Guo, R. Enen, and T. Mingxing, "A Hybrid Active Compensation Method for Current Balance Based on Y,d11 Connection Traction Transformer," Power Electronics and Intelligent Transportation System, 2008. PEITS '08. Workshop on. pp. 582-586, 2008.

[4] G. Ionescu, G. Paltanea, and V. Paltanea, "A method for minimization the harmonic distortions in three-phase inverter devices," 8th International Syposium on Advanced Topics in Electrical Engineering(ATEE). pp. $1-4,2013$.

[5] H. Mahmood and J. Jiang, "Modeling and Control System Design of a Grid Connected VSC Considering the Effect of the Interface Transformer Type," IEEE Transactions on Smart Grid, vol. 3, no. 1. pp. 122-134, 2012.

[6] F. Corcoles, L. Sainz, J. Pedra, J. SánchezNavarro, and M. Salichs, "Three-phase transformer modelling for unbalanced conditions, Part 1: core modelling and introductory examples," IET Electric Power Applications, vol. 2, no. 2. pp. 99-112, 2008.

[7] B. Singh, P. Jayaprakash, T. R. Somayajulu, and D. P. Kothari, "Reduced Rating VSC With a Zig-Zag Transformer for Current Compensation in a Three-Phase Four-Wire Distribution System," IEEE Trans. Power Deliv., vol. 24, no. 1, pp. 249-259, Jan. 2009.
[8] H.-L. Jou, K.-D. Wu, J.-C. Wu, and W.-J. Chiang, "A Three-Phase Four-Wire Power Filter Comprising a Three-Phase Three-Wire Active Power Filter and a Zig-Zag Transformer," IEEE Trans. Power Electron., vol. 23, no. 1, pp. 252-259, Jan. 2008.

[9] A. N. Arvindan and C. Sanal, "Investigation for harmonic mitigation in the line and neutral currents of three-phase four-wire system feeding two-pulse rectifiers with balanced and unbalanced load using Zig-Zag transformer," in 2014 IEEE 2nd International Conference on Electrical Energy Systems (ICEES), 2014, pp. 194-201.

[10] Sewan Choi and Minsoo Jang, "Analysis and Control of a Single-Phase-Inverter-ZigzagTransformer Hybrid Neutral-Current Suppressor in Three-Phase Four-Wire Systems," IEEE Trans. Ind. Electron., vol. 54, no. 4, pp. 2201-2208, Aug. 2007.

[11] P. S. C. Nair and P. P. K., "A novel method for recovery of drainage power from distribution transformers," Innovative Smart Grid Technologies (ISGT Europe), 2011 2nd IEEE PES International Conference and Exhibition on. pp. 1-5, 2011.

[12] K. Jin and C. Liu, "A Novel PWM High Voltage Conversion Ratio Bidirectional Three-Phase DC/DC Converter With Y- $\Delta$ Connected Transformer," IEEE Transactions on Power Electronics, vol. 31, no. 1. pp. 8188, 2016.

[13] L. Gu, K. Jin, and C. Liu, "Current-triplerrectifier pulse width modulation ZVS threephase full-bridge DC/DC converter with $\mathrm{Y}-\Delta$ connected transformer," IET Power Electronics, vol. 8, no. 7. pp. 1111-1118, 2015.

[14] R. Anuraj, A. Sathesh, and S. Smys, "Neutral current and harmonic mitigation using ZSBR with various transformer topologies," in 2015 2nd International Conference on Electronics and Communication Systems (ICECS), 2015, pp. $1695-1700$.

[15] E. Unicrom, "Polaridad de un transformador eléctrico," 2015. [Online]. Available: http://unicrom.com/polaridad-de-un-

transformador-electrico/. [Accessed: 19-Jun2016]. 